\title{
PULSE WAVE SHAPE ANALYSIS OF THE CARDIOVASCULAR SYSTEM USING HIGH SIGNAL RESOLUTION
}

\author{
ANNA SZYMIL ${ }^{1}$, RYSZARD KRZYMINIEWSKI ${ }^{1}$, BERNADETA DOBOSZ ${ }^{1}$, ADAM PAJĄK ${ }^{2}$, \\ ANDRZEJ SZYSZKA ${ }^{2}$, MALGORZATA LADZIŃSKA ${ }^{1}$ \\ ${ }^{1}$ A. Mickiewicz University, Faculty of Physics, Umultowska 85, Poznań 61-614 Poland \\ ${ }^{2}$ University of Medical Sciences, Second Department of Cardiology, 28 Czerwca 1956 roku, Poznań, 61-485 Poland
}

Received June 16, 2014; accepted August 29, 2014; published online September 4, 2014

\begin{abstract}
The aim of this study was to carry out the FMD (flow - mediated dilatation) and NID (nitroglycerin-induced dilatation) tests in the case of patients with hypertension by using new measurement method - High Signal Resolution Pulse Wave (HSR PW). The paper presents new diagnostic method HSR PW which was used to evaluate the pulse wave in patients with arterial hypertension. HSR-PW is a method based on increasing the resolution of the pulse wave signal, recorded during a standard test using the Fourier Transform. The study involved twenty-four patients with hypertension and fifteen healthy people as a control group. The analysis showed that changes of the shape of the pulse wave in HSR PW took place in both groups conducting the FMD and NID tests. The HSR PW method proved to be more sensitive to changes in the body then the standard pulse oximeter and therefore may increase clinical practicability.
\end{abstract}

\section{INTRODUCTION}

The arterial hypertension is the most common disease of the cardiovascular system in the World (Krasinska, Tykarski 2002). Due to the lack of distinct symptoms, which force the patient to seek medical care, it remains undiagnosed for a long time (Zdrojewski, Wyrzykowski, Szczech, Wierucki, Naruszewicz, Narkiewicz, 2005). At the same time the undiagnosed disorder slowly leads to the dysfunction of many vital organs (Kaplan, 2010). Early detection of these minimal changes is essential in order to introduce a suitable therapy which can stop their progression or even lead to regression (Egan, Zhao, Axon, 2010).

The influence of hypertension on the endothelium and its dysfunction has been widely described (Dominiczak, Bohr, 1995; Panza, Casino, Kilcoyne, Quyyumi, 1993). The proper functioning of the endothelium enables the maintenance of vessel homeostasis. The endothelium produces and releases substances into the body which influence coagulation and fibrinolysis and also vasodilatation (Verma, Anderson, 2002; Bonetti, Lerman, 2003; Tassorellis, Joseph, Buzzi, Nappi, 1999). The most important factor is Nitric oxide (NO) with rapid release plays a crucial role in reactivity of blood vessels (Moncada, Higgs, 1993; Neubauer-Geryk, Bieniaszewski, 2007; Tiefenbacher, 2010; Vita, Keaney, 2002).

The commonly applied method to measure endothelial function is the FMD (flow - mediated dilatation). It allows the assessment of the artery vasodilation, based on previous occlusion of the mentioned artery and reflects endothelial functions changes (Neubauer-Geryk, 2007). Brachial artery FMD has been shown to correlate with measures of coronary endothelial function (Anderson, Uehata, Gerhard, 1995)

The arterial dilatation may also be caused by the influence of nitroglycerin on the smooth muscle tissue of the vessels. Nitroglycerin-induced dilatation (NID) facilitates to estimate arterial dilatation regardless of the endothelial function (Neubauer-Geryk, 2007). Nitroglycerin relaxes blood vessels due to the release of the nitric oxide (Tiefenbacher, 2010). Moreover, the nitroglycerin has profound effects on the system resistance by lowering the systolic and diastolic arterial pressure and the stroke volume (Rojek, Szczech, 2006; Soderstrom, Sellgren, Ponten, 1999; Balinski, 2007).

Both FMD and NID of the brachial artery are related to cardiovascular risk factors (Adams, Robinson, McCredie, Seale, Sorensen, Deanfield, Celermajer, 1998). Early determination of endothelial function allows early detection of arterial and organ damage (Gomulka, Mizia-Stec, Gasior, Mizia, 2005; Perticone, Ceravolo, Pujia, 2001). These disorders can be observed using ultrasound sensor during FMD and NID tests. The pulse wave shape does not change in standard pulse oximeter records. For the first time to assess endothelial dysfunction, two simple provocation tests were used: 
FMD and NID by High Signal Resolution Pulse Wave (HSR PW).

The aim of this study was to carry out the FMD and NID tests in the case of patients with hypertension by using new measurement method - HSR PW.

\section{METHODS}

During vasodilatation tests the shape of the pulse wave was registered. Each patient had a pulse oximeter on the left hand index finger. A standard CMS-50E digital pulse oximeter, attached to the computer, was used to record the pulse waves. This method is quick, as it only takes few seconds and noninvasive.

HSR-PW is a new method invented by the Faculty of Physics at Adam Mickiewicz University in Poznań. It is used to increase the digital resolution of waveform from a standard record. To achieve this, the HSR method applies the linear transformation. This helps to obtain a more accurate analysis of the received signal. In contrast to the results of a standard measurement, even small changes in the shape of the signal can be easily recognized (Krzyminiewski, 2012; Krzyminiewski, Dobosz, Ladzinska, Jemielity, Buczkowski, Urbanowicz, 2011).

In the HSR pulse oximetry the linear transformation of the digital waveform pulse is used. The signal, obtained during the test, can be shown in the equation (1):

$$
F(x)=\int_{-\infty}^{\infty} K\left(x-x^{\prime}\right) \Psi\left(x^{\prime}\right) d x^{l},
$$

where the shape and width of spectral lines is described by a function $\mathrm{K}$ (where $\mathrm{K}$ is Gaussian function with $0,1 \mathrm{~ms}$ width), while $\Psi$ is a function which describes the positions and intensities of individual components of the signal. To determine the $\Psi$ function the $\mathrm{F}$ function is transformed using the Fourier Transform (Madisetti,
1998; Krzyminiewski 1999). The following equation (2) can be obtained:

$$
\widetilde{F}(y)=\frac{1}{\sqrt{2 \pi}} \int_{-\infty}^{\infty} F(x) \exp (-i x y) d x
$$

On the basis of the equation above, $\Psi(\mathrm{x})$ function can be determined, equation (3):

$$
\widetilde{\Psi}(x)=\frac{1}{\sqrt{2 \pi}} \int_{-\infty}^{\infty} \frac{\widetilde{F}(y)}{\widetilde{K}(y)} \exp (i x y) d y \text {. }
$$

The effect of increasing the signal resolution procedures on the pulse wave recorded by the use of pulse oximeter is shown in Fig. 1. Implementation of the linear transformation of the signal increases the accuracy of peaks' separation (Krzyminiewski, 2012; Krzyminiewski, et al., 2011a).

The focus group of more than four hundred persons, tested by the Faculty of Physics, Medical Physics Division of Adam Mickiewicz University in Poznań, allows the obtainment of the average pulse wave and determination of its standard parameters (Krzyminiewski, Stepien, Dobosz, 2011b). Apart from the standard data, such as pulse and saturation, the HSR PW test allows to determine additional parameters describing the shape of the pulse wave. For example: $\mathrm{K} 1 / \mathrm{K} 2$ - is the ratio of amplitude of two first maximal peaks, A1/AV - the amplitude of the first minimum to the average value of the single evolution of the high definition pulse wave, V/A - (Ventricle/Aorta) is calculated as the ratio of the surfaces defined by equation (4) and presented in Fig. 2:

$$
V / A=\frac{s_{1}+s_{2}}{s_{1}}
$$

Factors used in calculation are shown in Fig. 2.

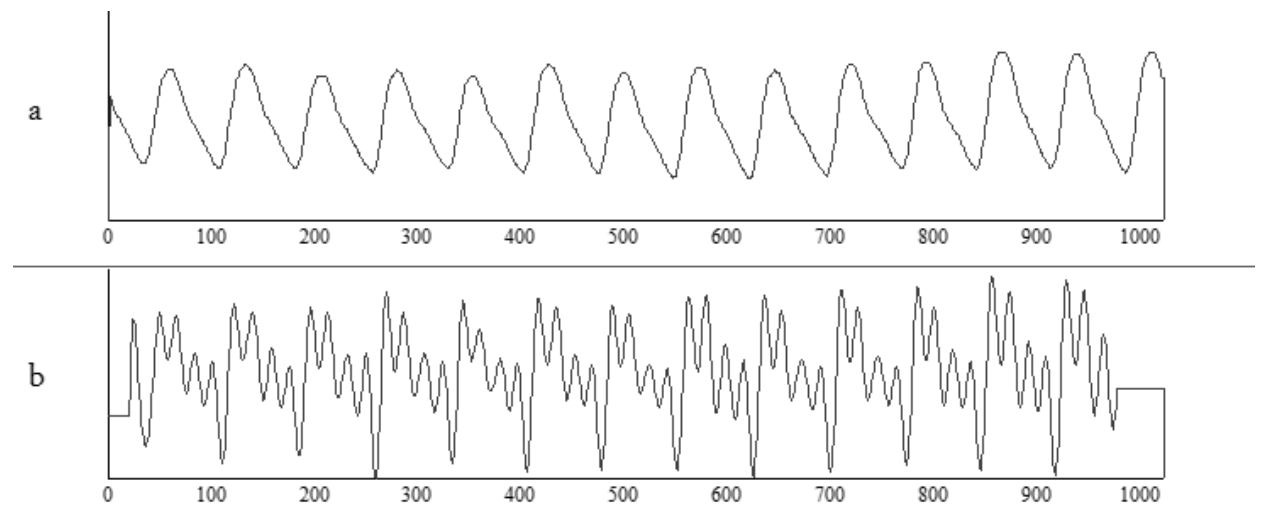

Fig. 1. Pulse wave recorded with pulse oximeter: a) The standard record, b) Record after HSR PW analysis. 


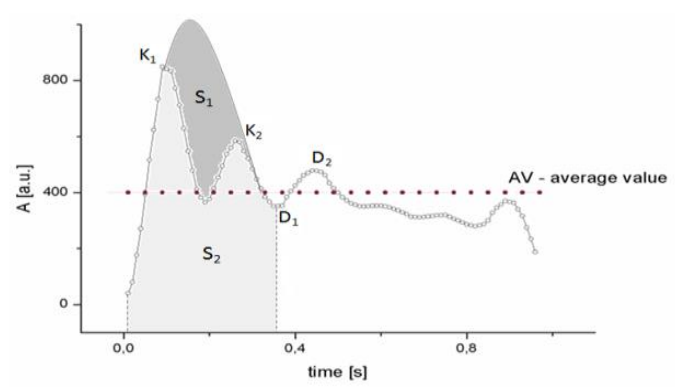

Fig. 2. Labelling of amplitudes of appropriate maximal and minimal values of HSR PW.

One of the most sensitive HSR PW parameters is $\mathrm{K} 1 / \mathrm{K} 2$ which value in healthy persons is bigger than 1 . Disorders of this parameter can be observed in the case of aortic valve replacement procedure. The results, obtained before the surgery, were entirely different than the pulse wave of a healthy person, which could not be observed by the use of the standard pulse oximetry test (Fig. 3). After the procedure the shape of the pulse wave as well as the $\mathrm{K} 1 / \mathrm{K} 2$ parameter were back to normal (Krzyminiewski, et al., 2011b).

An exemplary pulse oximetry HRS test carried out before the aortic valve replacement procedure, as well as after the operation is presented in the Fig. 3.

In order to demonstrate that the value of the parameter $\mathrm{K} 1 / \mathrm{K} 2$ is determined by, among others, vascular resistance, an experiment was carried out. For this purpose, when recording the pulse wave using the pulse oximeter, compression of palmar arch arteries was performed. Fig. 4 shows the change in shape of the pulse wave during the experiment.

The results obtained indicate that the change in shape of the pulse wave at the moment of compression is not the effect of reflected wave. The ratio of the parameter $\mathrm{K} 1 / \mathrm{K} 2$ is determined by vascular resistance. a)

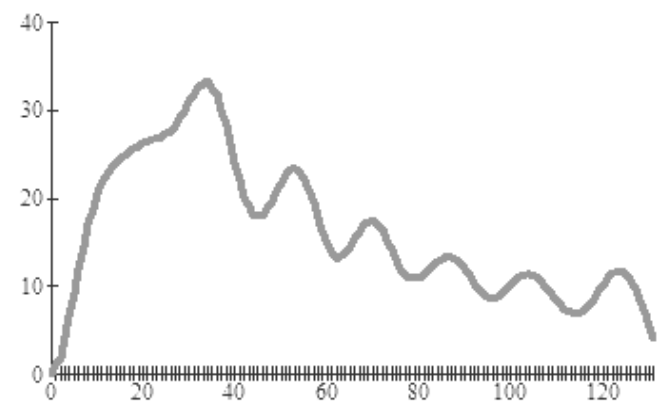

b)

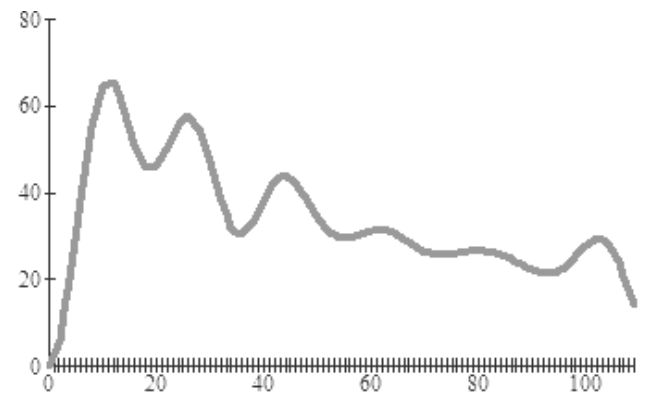

Fig. 3. HSR pulse wave analysis of the same patient before a) and after b) aortic valve surgery.

Furthermore, the HSR PW allows the determination of the pulsatility index $(P I)$, which is calculated as in the case of the Doppler test, in accordance with the equation (5), (Fornal, Janicki, Grodzicki, 2003):

$$
P I=\frac{A_{\max }-A_{\min }}{A V} .
$$

$A_{\max }$ - maximum velocity (in HSR-PW maximum amplitude), $A_{\min }-$ minimum velocity (in HSR-PW minimum amplitude), $A V$ - average velocity (in HSRPW average amplitude).

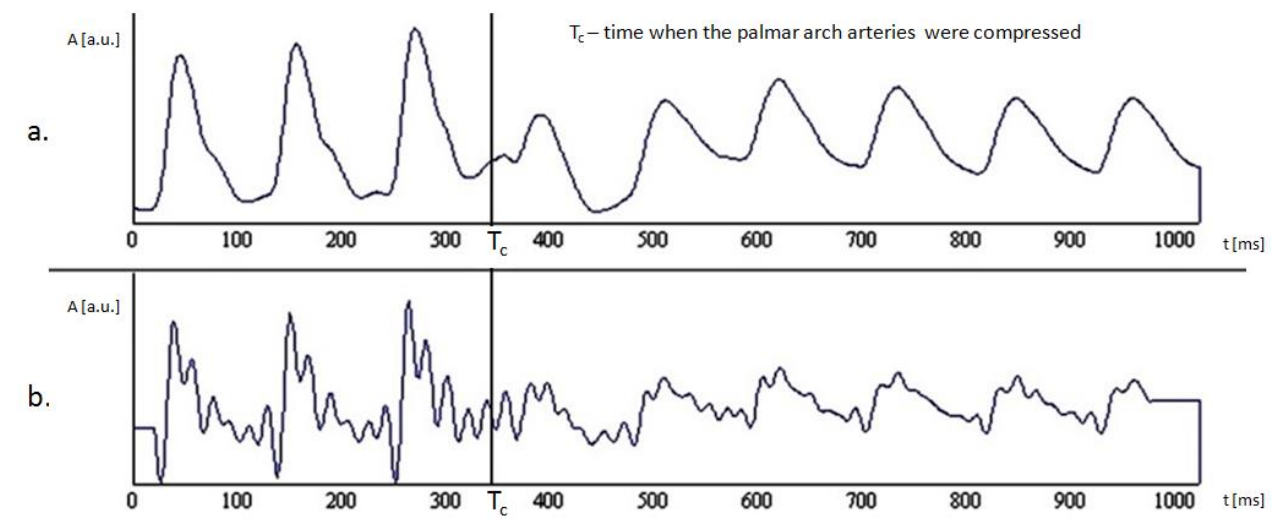

Fig. 4. Pulse wave recorded with pulse oximeter when the compression of palmar arch arteries was performed: a) The standard record, b) Record after HSR PW analysis. 
Contemporary literature provides several models, which describe the processes responsible for the creation of the pulse wave. There are two widely described models in literature. One of them takes into consideration the wave reflected from resistance vessels (Rajzer, Kawecka-Jaszcz, 2002) and the second is the Windkessel model (Nichols, O'Rourke, 1990; Kind, Faes, Vonk-Noordegraaf, Verhaegen, 2010; Irace, Fiaschi, Cortese, Gnasso, 2006).

The first model takes into account the creation of two waves: wave reflected from resistance vessels and the wave coming from the heart. The first mentioned corresponds to the arterial pressure and its size is covered by the arterial stiffness and the distance from the place of reflection (Rajzer, 2002; Nichols 1990). The second is influenced by the stroke volume and arterial stiffness. The arterial pressure waveform also depends on the heart rate and heart pumping dynamics.

The Windkessel model presents the heart as a suction pump, whereas the function of elastic vessels is illustrated by an air-chamber, filled with compressed gas. A non-elastic hose, connected to the air-chamber, corresponds to the resistance vessels. The Windkessel model correctly reflects the problems with complete susceptibility of arteries and peripheral resistance (Kind, 2010).

Currently, the interpretation of particular HSR PW peaks shape is at trial stage. So far the results lead to the suggestion, that the ratio of $\mathrm{K} 1 / \mathrm{K} 2$ amplitudes characterizes the dynamics of the stroke volume from the left ventricle, as well as the functioning of the aortal valve and vascular resistance. Changes of the shape and $\mathrm{K} 1 / \mathrm{K} 2$ relations were confirmed in tests of the pulse wave in the case of patients with fault aortal valve. The obtained results are presented in the Fig. 3 (Krzyminiewski, 2011b). V/A parameter inform about the aorta's susceptibility to the blood pressure change, caused by the cramp of the left ventricle, whereas D1 and D2 peaks are responsible for dichotic notch, which represents the closing of the aortal valve and constitutes the beginning of the ventricular diastole. Next peaks are related to modifications of the pulse waves found from the moment of its creation to the registration place, illustrating the elastic function of the peripheral vessels. Such an interpretation corresponds to the provisions of the Windkessel model. The participation of the reflected wave, a fact confirmed in previous tests, does not influence the shape of the pulse wave measured at the finger, the end of the artery (Nichols, 1990). The obtained results indicate that the HSR pulse oximetry method is definitely more sensitive than the standard pulse oximetry test (Krzyminiewski, 2012; Krzyminiewski, Szymił, Dobosz, Majewski, 2011c).

In the case of the hypertension initial stadium there is lack of clinical symptoms. The slow and destructive process causes endothelial dysfunction (Dominiczak et al., 1995; Panza et al., 1993). Due to its unquestionably higher measurement sensitivity, the HSR PW may appear to be an excellent diagnostic tool.

\section{MATERIALS}

The study was approved by the hospital's ethical committee, and each patient. Twenty-four patients diagnosed with hypertension (at least ten years after the diagnosis) were included into the research. Patients involved in the study of the Second Department of Cardiology in Poznań underwent two vasodilatation tests FMD and NID. In order to compare the results, both tests were additionally performed on the control group of fifteen people. Patients were entered into the study at the time of an optimal alignment of their medical status. The test consisted of three parts: the analysis of the resting pulse wave, the flow-induced vasodilatation FMD and the nitroglycerin-induced vasodilatation tests NID.

Twelve hours prior to the tests the patients were asked to avoid the consumption of caffeine, alcohol and cigarettes. Measurements were made in a quiet room, with the patients sitting on a chair. A cuff, at the time of the flow-induced vasodilatation test, was entwined around the forearm, approximately $5 \mathrm{~cm}$ below the cubital fossa. The measured systolic blood pressure, to which the cuff was inflated, was $50 \mathrm{mmHg}$ higher compared to the actual patient's pressure. The ischemic phase lasted three minutes, after which the cuff was rapidly deflated. The measurement of pulse wave shape was executed before mounting the cuff, then immediately (up to 15 seconds) after deflation and sequentially in the second minute. Then after a tenminute rest in a sitting position the next NID test was performed. After the exclusion of contraindications the patient was given 400ug nitroglycerin spray (Nitromint) sublingually. The evaluation of the pulse wave was performed prior to administration, and then, two and fourth minutes after. At the same time the blood pressure was measured.

All variables received from the test were illustrated as the average $\pm \mathrm{SD}$ as well as a comparison with the application of t-Student test. All analyses were carried out with the application of Statistica programme, version 6.0. The relevance level during the test analysis amounted to $\alpha=0,05$.

\section{RESULTS}

The FMD results obtained for both groups are illustrated in table 1. The analysis took into account two parameters (K1/K2 and A1/AV) determined upon the basis of the HSR PW. The average value of K1/K2 
Table 1. Results of FMD test in hypertension $(\mathrm{H})$ and control group $(\mathrm{C})$.

\begin{tabular}{lccccc}
\hline $\begin{array}{l}\text { HSR PW } \\
\text { parameters }\end{array}$ & \multicolumn{2}{c}{ K1/K2 } & \multicolumn{3}{c}{ A1/AV } \\
Group & $\mathrm{H}$ & $\mathrm{C}$ & $\mathrm{P}$ & $\mathrm{H}$ & $\mathrm{C}$ \\
\hline $\begin{array}{l}\text { Before } \\
\begin{array}{l}\text { 15s after } \\
\text { deflation }\end{array}\end{array}$ & $1,11 \pm 0,24$ & $1,44 \pm 0,24$ & $\mathrm{p}<0,05$ & $1,39 \pm 0,24$ & $0,80 \pm 0,1$ \\
$\begin{array}{l}\text { 120s after } \\
\text { deflation }\end{array}$ & $1,00 \pm 0,21$ & $1,05 \pm 0,24$ & & $1,35 \pm 0,19$ & $1,13 \pm 0,18$ \\
\hline
\end{tabular}

parameter before the commencement of the FMD test for the group, suffering from hypertension, amounted to $1,11_{ \pm} 0,24$, whereas in the case of the control group to $1,44 \pm 0,24$, hence constituting approximately $23 \%$ of difference $(p<0,05)$. Fifteen seconds after the cuff deflation, the value decreased definitely, i.e. with $27 \%$, in the case of the control group and, in the case of the group suffering from hypertension it decreased, only by $10 \%$. Two minutes after the deflation for the hypertension group this parameter was still differing from the value taken before the test $(\mathrm{p}=0,029)$, such as in the control group $(p=0,934)$. In the case of the additionally analyzed parameter A1/AV, the average value in the hypertension group amounted to $1,39_{ \pm} 0,24$, but, in the case of the control group, it was $42 \%$ lower, i.e. $0,80_{ \pm} 0,1(\mathrm{p}<0,05)$. Upon 120 seconds this parameter for the group suffering from hypertension was still significantly differing, with regard to statistics, compared to the value of the earlier performed test $(p=0,013)$, whereas in the control group it returned to its previous value $(p=0,065)$ (Tab. 1). The factor V/A has been omitted due to the lack of significant differences with regard to statistics. Example of pulse wave for FMD test in both groups is shown in Fig. 5.

a)

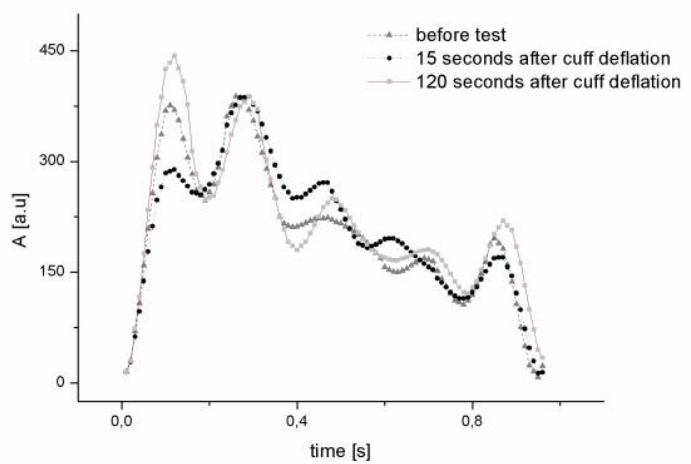

b)

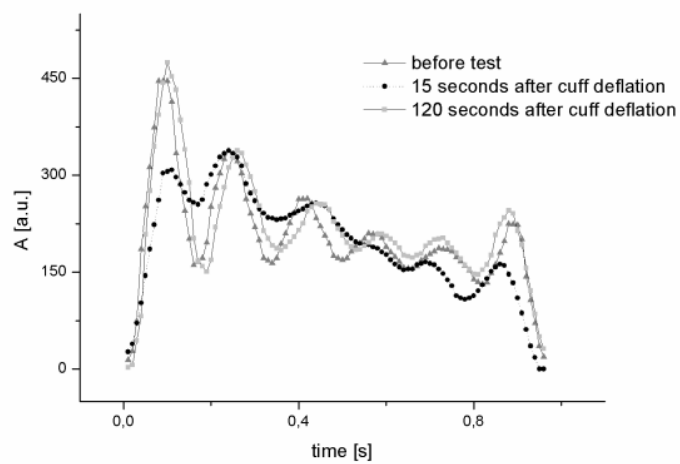

Fig. 5. Pulse wave after HSR PW analysis in the FMD test; a) patients with arterial hypertension; b) control group.

Table 2. The NID test results: the average value (AV), pulsatility index (PI), blood pressures: systolic (Ps), diastolic ( $\mathrm{Pd})$ and pulse before and after nitroglycerin administration in hypertension $(\mathrm{H})$ and control group $(\mathrm{C})$.

\begin{tabular}{lcccc}
\hline & \multicolumn{2}{c}{$\mathrm{H}$} & \multicolumn{2}{c}{$\mathrm{C}$} \\
& Before & After & Before & After \\
\hline AV [a.u.] & $230 \pm 60$ & $200 \pm 50$ & $230 \pm 70$ & $220 \pm 60$ \\
PI [a.u.] & $1,88 \pm 0,30$ & $2,12 \pm 0,14$ & $2,06 \pm 0,11$ & $2,23 \pm 0,18$ \\
Pulse & & & & \\
{$[\mathrm{BPM}]$} & $71 \pm 9$ & $85 \pm 15$ & $79 \pm 14$ & $98 \pm 18$ \\
Ps & & & & \\
{$[\mathrm{mmHg}]$} & $140 \pm 19$ & $121 \pm 17$ & $113 \pm 9$ & $71 \pm 7$ \\
Pd & & & & \\
{$[\mathrm{mmHg}]$} & $81 \pm 11$ & $76 \pm 12$ & $105 \pm 14$ & $67 \pm 7$ \\
\hline
\end{tabular}

Based upon the HSP PW for the NID test, three parameters describing the shape of the pulse wave were specified. The average value of the $\mathrm{K} 1 / \mathrm{K} 2$ parameter upon 2 minutes from the application of the NTG under the tongue, was definitely higher in both tested groups, i.e. $35 \%$ (hypertension group) and $26 \%$ (control group). Within the next 2 minutes the parameter did not change significantly with regard to statistics than the value gathered from the test conducted 2 minutes before on both groups. After 120 seconds the value of the second 
Table 3. The NID test results before and after nitroglycerin administration in the hypertension (H) and control group (C) in the NID test.

\begin{tabular}{lccccccccc}
\hline $\begin{array}{l}\text { HSR PW } \\
\text { parameters } \\
\text { Group }\end{array}$ & \multicolumn{3}{c}{ K1/K2 } & \multicolumn{3}{c}{ A1/AV } & \multicolumn{3}{c}{ V/A } \\
\hline Before & $1,16 \pm 0,32$ & $1,49 \pm 0,20$ & $\mathrm{p}=0,01$ & $1,35 \pm 0,23$ & $0,86 \pm 0,14$ & $\mathrm{p}<0,05$ & $8,2 \pm 3,9$ & $3,5 \pm 0,4$ & $\mathrm{p}<0,05$ \\
After 120s & $1,8 \pm 0,4$ & $2,02 \pm 0,22$ & & $1,16 \pm 0,22$ & $0,96 \pm 0,10$ & & $5,3 \pm 2,4$ & $3,9 \pm 0,6$ & $\mathrm{C}$ \\
After 240s & $1,9 \pm 0,5$ & $2,00 \pm 0,23$ & & $1,09 \pm 0,25$ & $0,90 \pm 0,12$ & & $5,0 \pm 2,7$ & $5,2 \pm 0,5$ \\
\hline
\end{tabular}

analyzed parameter (A1/AV), in the case of hypertension group, was $1,16_{ \pm} 0,22$ and after 240 seconds only $1,09_{ \pm} 0,25$ and therefore still significantly different with regard to statistics than the value collected before the test $(\mathrm{p}<0,05)$. In the case of the control group, no significant differences with regard to statistics could be extracted. The last analyzed parameter was the V/A relation, which in the hypertension group was placed definitely beyond the norm and differed by almost $43 \%$ in respect to the average value in the control group before the application of the medicine. 4 minutes after the application of the nitroglycerin the value of V/A parameter decreased in the hypertension group $(39 \%)$ and increased in the control group (48\%) compared to the average value taken before the application of the medicine (Tab. 3). Additionally, the NID test involved the determination of the average value (AV) and the pulsatility index (PI) based upon the high definition pulse wave in accordance with the formula (5). The average value of the high definition pulse wave before and after the application of nitroglycerin in both groups was significantly different with regard to statistics (both groups $p<0,05)$. The pulsatility index also showed the same tendency - both groups $\mathrm{p}<0,05$. The results were illustrated in Tab. 2. An example of pulse waveform for the NID test in both groups shows Fig. 6.

In order to determine the time of returning of the $\mathrm{K} 1 / \mathrm{K} 2$ parameter to the value taken before the medicine administration a series of measurements of the pulse wave was carried out. The diagram of the aforementioned relation is illustrated in the Fig. 7. The line, which joins the measurement points, was crossed out in order to facilitate data tracking. a)

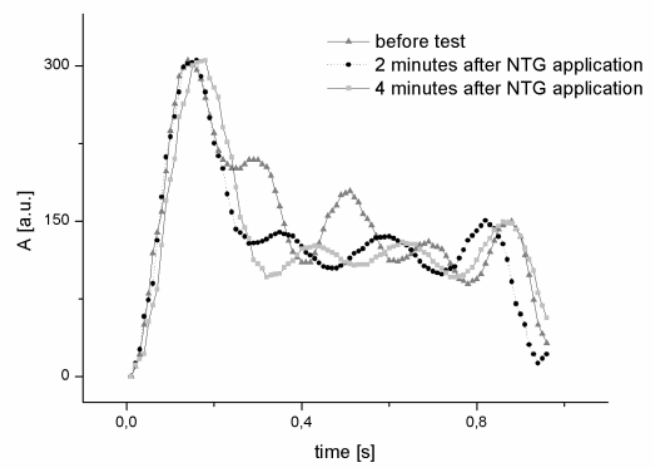

b)

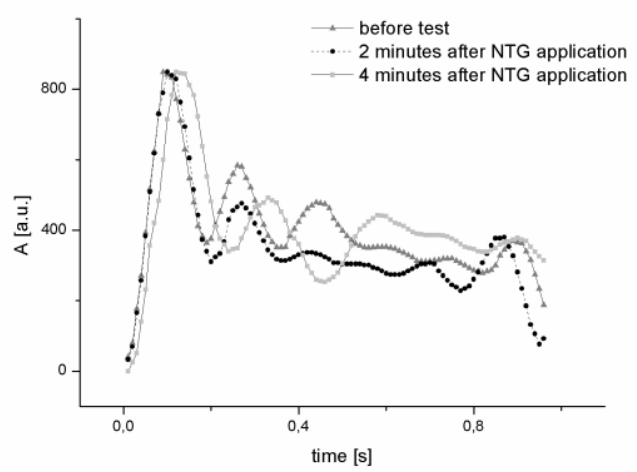

Fig. 6. Pulse wave after HSR PW analysis in the NID test: a) patients with arterial hypertension; b) control group. 


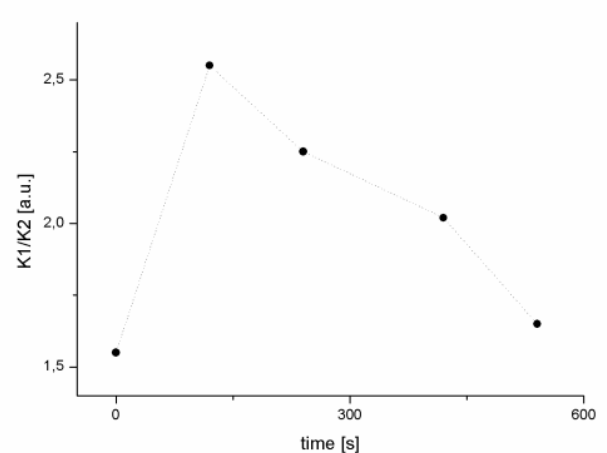

Fig. 7. Changes of K1/K2 parameter over time.

\section{CONCLUSION}

The new measurement method HSR PW, applied for the first time in the FMD (flow - mediated dilatation) and NID (nitroglycerin-induced dilatation) tests, proved to be a highly sensitive for the detection of vascular resistance and also simple in its application. The received record of the pulse wave after the HSR PW analysis showed significant changes, which could not be observed by the use of the standard pulse oximetry. The new HSR PW method helped to distinguish people with hypertension from healthy people. For example the average of parameter $\mathrm{K} 1 / \mathrm{K} 2$ for the group, suffering from hypertension, amounted to $1,11_{ \pm} 0,24$, whereas in the case of the control group to $1,44_{ \pm} 0,24$, (23\% of difference) also parameter $\mathrm{A} 1 / \mathrm{AV}$ in the hypertension group was $1,39_{ \pm} 0,24$, but in the case of the control group, it was $42 \%$ lower.

In addition the applied modelling satisfactorily allows describing the changes of the vessel resistance in the conducted FMD and NID tests. The obtained results provide a good basis for further tests, which may introduce an important element into the complex diagnostics of the circulatory system.

\section{REFERENCES}

Krasinska B., Tykarski A. (2002) The influence of hypotensive drugs on the arterial compliance in hypertension, Medical News; 71: 176-83.

Zdrojewski, T., Wyrzykowski B., Szczech R., Wierucki L., Naruszewicz M., Narkiewicz K., (2005), Epidemiology and prevention of arterial hypertension in Poland. Blood Press Suppl; 2: 10-6.

Kaplan N. M. (2005), Kaplan's Clinical Hypertension, Lippincott Wiliams and Wilkins, 5.

Egan BM, Zhao Y, Axon RN (2010). US trends in prevalence, awareness, treatment, and control of hypertension 19882008. JAMA; 26:2043-50
Dominiczak AF., Bohr DF. (1995), Nitric oxide and its putative role in hypertension. Hypertension; 25: 1202-11

Panza JA., Casino PR., Kilcoyne CM., Quyyumi AA. (1993), Role of endothelium-derived nitric oxide in the abnormal endothelium-dependent vascular relaxation of patients with essential hypertension, Circulation; 87:1468-74.

Verma S., Anderson T.J. (2002): Fundamentals of endothelial function for the clinical cardiologist. Circulation; 105: 54652.

Bonetti P.O.,Lerman A. (2003): Endothelial dysfunction. A marker of atherosclerotic risk. Arterioscler. Thromb. Vasc. Biol.; 23: 168-78.

Tassorellis C., Joseph S.A., Buzzi M.G., Nappi G. (1999) The effects on the central nervous system of nitroglycerin putative mechanisms and mediators. Progress in Neurobiology; 57:607-24.

Moncada S, Higgs A. (1993), The L-arginine-nitric oxide pathway. N Engl J Med.; 329:2002-12.

Neubauer-Geryk J., Bieniaszewski L. (2007), Methods of assessing endothelium. Vasodilatation of brachial artery after ischemia. Heart and Vascular Diseases; 4.4:190-96.

Tiefenbacher CP. (2001) Tetrahydrobiopterin: A critical cofactor for eNOS and a strategy in the treatment of endothelial dysfunction? Heart and Circulatory Physiology; 280:2484-88.

Vita M.J., Keaney J.F. (2002), Endothelial function a barometer for cardiovascular risk? Circulation; 106:640-46.

Anderson T.J., Uehata A., Gerhard M.D., et al. (1995), Close relationship of endothelial function in the human coronary and peripheral circulations. J Am Coll Cardiol.;26:1235-41

Rojek A., Szczech R.( 2006), The side effests of nitroglycerin - need for education patients. Heart and Vascular Diseases; 3.4: $187-92$

Soderstrom S., Sellgren J., Ponten J. (1999), Aortic and Radial Pulse Contour: Different Effects of Nitroglycerin and Prostacyclin. Anesth Analg; 89:566-72

Balinski M. (2007), Score autonomic modulation of the cardiovascular system, vascular stiffness and peripheral perfusion after nitroglycerin administration in healthy subjects, $19-25$

Adams M.R., Robinson J., McCredie R., Seale J.P., Sorensen K.E., Deanfield J.E., Celermajer D.S. (1998), Smooth muscle dysfunction occurs independently of impaired endothelium-dependent dilation in adults at risk of atherosclerosis. J Am Coll Cardiol;32 :123-27.

Gomulka S., Mizia-Stec K., Gasior Z., Mizia M. (2005) Noninvasive assessment of endothelial function. The Chair of Cardiology 7,1:77-82

Perticone F., Ceravolo R., Pujia A. (2001) Prognostic significance of endothelial dysfunction in hypertensive patients. Circulation; 104: 191-96.

Krzyminiewski R. (2012), TelMedHome - fast and cheap home care monitoring of the patients with cardiac diseases, InM Jordanova and F. Lievens (Eds)Global Telemedicine and eHealth Updates: Knowledge reasources, Pub ISfTeH, Luxemburg Vol. 5, pp 623-627.

Krzyminiewski R., Dobosz B., Ladzinska M., Jemielity M., Buczkowski P., Urbanowicz T., et al., (2011a) High Signal Resolution Pulse Wave - Hope for a Fast and Cheaop Home Care Monitoriing Patients with Cardiac Diseases, Mede-Tel Conference Proceedings; 252-56. 
Madisetti V.K. (1998), The digital signal processing handbook, Douglas B. Williams IEEEpRess.

Krzyminiewski R. (1994), Computer enhancement of complex spectroscopic spectra resolution, Mel Phes Reports, 6, 17480.

Krzyminiewski R., Stepien R., Dobosz B. (2011b), Correlations between high signal resolution pulsoximeter parameters and artery stiffness of patients with atherosclerosis. Polish Journal of Medical Physics and engineering; 17: 16.

Fornal M., Janicki K., Grodzicki T. (2003), Effest of nitrates on blond flow in carotid arteries. Folia Cardiologica; 10.3: 347-51.

Rajzer M., Kawecka-Jaszcz K., (2002) Arterial compliance in arterial hypertension. From pathophysiology to clinical relevance. Arterial Hypertension; 6.1: 61-73.

Nichols W.W., O'Rourke M.F. McDonald's Blood Flow in Arteries. 3rd ed. Philadelphia, Pa: Lea \& Febiger; 1990: 216-50.

Kind T. Faes, T.J.C., J.W., Vonk-Noordegraaf, A., Verhaegen M. (2010), Estimation of Three- and Four-Element Windkessel Parameters Using Subspace Model Identification. Biomedical Engineering; 57. 7: 1531 - 38.

Matthys K. S., Alastruey J., Peiro J., Khir A. W., Segers P., Verdonck P. R., Parker K. H., Sherwin S. J. (2007) Pulse wave propagation in a model human arterial network: Assessment of 1-D numerical simulations against in vitro measurements. Journal of Biomechanics; 40: 3476-86.

Krzyminiewski R., Szymił A., Dobosz B., Majewski M. (2011c), HSR-PW as a method of patient monitoring during and after short intravenous anesthesia. Polish Journal of Medical Physics and engineering; 17: 113.

Irace C., Fiaschi E., Cortese C., Gnasso A. (2006) Flowmediated vasodilatation on the brachial artery and intimamedia thickness of carotid artery in never-treated subjects. Int. Angiel.;25: 274-79 